\title{
CORRIGENDUM
}

\section{On isotropic tensors}

\section{SIR HAROLD JEFFREYS}

(1) Proc. Cambridge Philos. Soc. 73 (1973), page 173

Dr E. Leibowitz has called my attention to a mistake in my paper on isotropic tensors. (1) On p. 173, if $l_{12}$ is annihilated as stated and then we annihilate $l_{13}$ by the same method, a new contribution to $l_{12}$ appears. The correct method is to choose $\alpha$ so as to annihilate $l_{21}$. Then a suitable rotation on the 13 plane removes $l_{31}$ without reintroducing $l_{21}$. The rest of the argument stands. 